\title{
Criteria of Patient Selection in a Clinical Trial
}

\author{
Michel Bourin* \\ Neurobiology of mood disorders University of Nantes, France
}

Received: April 6, 2017; Accepted: May 30, 2017; Published: June 7, 2017

*Corresponding author: Michel Bourin, Neurobiology of mood disorders University of Nantes, 98, rue Joseph Blanchart, 44100, Nantes, France, E-mail: michel.bourin@univ-nantes.fr

\begin{abstract}
Which criteria will govern patient selection if this choice is to take various requirements into account and if the key problem is to obtain homogeneous groups in order to reduce variability in response and yet provide representative samples of the affection studied? The means of selecting patients will be defined by inclusion and exclusion criteria, which should be as explicit as possible.
\end{abstract}

Keywords: Clinical Trials; Selection Criteria; Patient Selection

\section{Introduction}

The selection of patients for a therapeutic trial involves many often contradictory requirements [1,2]. In particular, subgroups of patients are selected, whereas the drugs are intended for a large population. Moreover, rapid results are needed, which justifies the recruitment of patients with a high degree of homogeneity. Finally, the selection criteria should involve as few risks as possible for the patients. The contradiction is thus between the rigor required and the desire for representativeness.

The selection process is based on five requirements:

1.Isolating a group for which there is a greater or lesser chance of detecting a possible difference between the treatments compared;

2.Establishing a homogeneous group in order to reduce the variability of response, thus making statistical comparison more sensitive and decreasing the risk of bias due to the constitution of non-homogeneous groups;

3.Obtaining representative samples of the affection studied;

4.Defining the rules corresponding to realistic recruitment;

5.Respecting ethical obligations.

\section{General points}

The selection of patients for testing drugs is a very complicated process even overtly; subconscious bias must be avoided and our social conscience respected. It is of prime importance to define in advance exactly what you want to test and this may include not only the therapeutic and pharmacological effects and side-effects. Patient selection is a complicated process in which there is inevitably some bias and our job is to see that any biases which are included do not bias the results, and that the results are understood to apply to this particular selection of patients and not to any other. Too many explicit exclusions therefore make results less useful because less widely relevant [3].

As selection should favor measurement of the expected difference between two treatments, the objective needs to be defined. It may be to evaluate the variations in blood pressure caused by two antihypertensive agents regardless of their pharmacologic class, or to define the nosological situation if diuretics are studied in the context of arterial hypertension, or to delineate the clinical form clearly in order to determine whether it is symptomatic or progressive (an episode, complication or recurrence) or a matter of pathogenesis. Special attention should be paid to borderline cases which may involve important variations. In fact, everything needs to be considered, including the chances of success for the treatments compared (particularly the rapidity of cure).

\section{Selecting an Homogenous Group}

The patients selected should be likely to have a nearly comparable spontaneous course during the trial and especially a nearly identical sensitivity to the treatment compared. Otherwise, there is a risk that the groups will not be comparable, with a resulting variability of responses which would make the conclusions relatively unclear. Too vague a definition of the type of patients included would inevitably lead to a heterogeneous selection, providing discordant results and making the trial impossible to reproduce. Thus, it is advisable to determine the following points precisely: the nosological situation, the possibility of an associated pathology (comorbidity), and the social, demographic and, of course, physiologic characteristics which could influence the result [4].

The nosological situation, if defined precisely, should include an indication of symptom intensity. This will most often require numerical assessment, whether by laboratory examinations or scales (for psychiatry). A patient could thus satisfy the diagnostic criteria of DSM 5 relative to depression and only be included if his score on the Montgomery Asberg scale was more than 20. The evaluation of new drug entities with specific modes of action may be hampered by rigid diagnostic classification systems and patient selection processes that do not 
focus on the anticipated symptomatic, behavioral, and functional outcomes to be achieved. Patients enrolled in central nervous system (CNS) clinical trials may present with a heterogeneous group of symptoms representing several syndromes or subtypes, subsumed under the same diagnosis in the DSM 5 classification system [5]. As a result, enrolled patients may not have the valid illness characteristics of interest to the particular study. Clinical drug development needs to focus on the primary nosological entity likely to be affected by a new drug entity's mode of action. Ideally, a valid patient will have the acute primary symptoms that the novel drug is supposed to influence.

The same is true for intermediary criteria such as blood pressure which should be clearly determined. A concomitant pathology should be considered if it is likely to have an influence on the course of the disease, the judgment criteria and the metabolism of the drugs to be compared in the study. Thus, subjects at risk because of hepatic or renal insufficiency are often excluded from a trial if the drugs are eliminated mainly through the kidneys or if hepatic metabolism is an important factor. Finally, demographic, social and physiologic characteristics should also be considered for their possible influence on the results of the trial. This concerns essentially age, gender, weight and height, especially if the trial involves a fixed dosage. The patients should also understand the protocol, which may not be the case if there is a problem of language or of intellectual level in the event that self-evaluation scales are used.

\section{Selecting a Representative Sample of the Affection Studied}

Theoretically, the results of an experiment can be extrapolated if they relate to a representative sample of a hypothetical population corresponding to all of the patients with the pathology in question. Obviously, the sample is not truly representative since it does not depend on drawing lots from the total population. Moreover, it is not random since the physicians participating in the trial do not represent the entire profession, nor the individuals included the totality of patients. Finally, this sample excludes age categories as well as all comorbidities. In practical terms, a problem arises the other way around when an attempt is made a posteriori and empirically, on the basis of the patients included, to identify a pathological context theoretically corresponding to the population studied [6].

It is necessary in preliminary studies to envisage a sufficiently broad representation of a given pathological state and then, if possible, conduct studies on more homogeneous subgroups. In any case, it must be kept in mind that the requirements of homogeneity and representativeness are perfectly contradictory. A group is less representative to the extent that it is more homogeneous, and realistic compromises are not always possible.

\section{Ensuring a Realistic Recruitment}

Conducting clinical trial feasibility is one of the first steps in clinical trial conduct. This process includes assessing internal and environmental capacity, alignment of the clinical trial in terms of study design, dose of investigational product, comparator, patient type, with the local environment and assessing potential of conducting clinical trial in a specific country [7].

Recruitment to clinical trials remains a challenge, particularly in primary care settings. Initial projections of participant recruitment need to be as accurate as possible in order to avoid the financial, clinical and ethical costs of trial extensions or failures. However, estimation of recruitment rates is challenging and often poorly executed, if attempted at all. When an investigator is consulted about his recruitment practices in a given pathology, the replies are always overestimated. In fact, the problem is twofold: the pathology considered to be frequent is often less so, particularly at certain seasons, and the investigator more or less assumes that he will be able to recruit all the patients needed with this type of pathology.

In fact, the possibilities for recruitment are limited. The inclusion criteria may exclude subjects, for example, whose pathology is not exactly that desired or who present comorbidity. The exclusion criteria very often eliminate individuals not satisfying the age requirement. This overestimation is also related to the fact that some patients may refuse to enter the trial since it is not always easy to convince them of the notion of the placebo. Moreover, it is not reasonable to include a patient who is not well known by the investigator who, for example, is being seen for the first time. The recruitment procedure corresponds roughly to a very simple law, i.e. that only $10 \%$ of the patients with the pathology in question who are seen regularly by the investigator are likely to be included in the study.

A large number of factors that physicians felt impact recruitment rates to primary care research and highlighted the complexity of realistic estimation. Suitable early planning of the recruitment process is essential, and there may be potential to improve the projection of trial timelines by reducing biases involved in the process.

A good feasibility assessment looks to answer whether the study in question fits with the service provided for the patient group with whom the study is concerned. In addition it looks to assess how far above and beyond standard care the study related procedures or interventions are and how acceptable these are for patients, for example number of additional study visits, scans, blood tests etc. Other considerations at a local level should be around competing/conflicting studies that may affect the ability to recruit suitable participants and the practicalities for the day to day running of the study in relation to study personnel including numbers, expertise and availability of study team for the tasks required. The use of support services such as pharmacy, laboratories and contemplating of out of hours working (if applicable) should also be considered at this point [8].

\section{Respecting ethical considerations}

Much attention has been paid to the use of ethical principles to guide the conduct of clinical trials [9]. Less has been done to clarify and assess the "weights" assigned by clinicians 
(and others) to the values that come into conflict when patients are offered entry into trials. Quantitative techniques of value assessment were used to measure the relative importance of variables frequently identified as barriers to the entry of patients into clinical trials.

The strategy of randomized consent to facilitate the cooperation of eligible patients has been tried on a number of occasions, but its role is limited by the small proportion of patients who refuse to participate relative to the proportion for whom the decision not to participate is made by the physician

Informed consent is vital to the conduct of clinical trials to assure institutional review bodies that all precautions have been taken to inform patients before they are enrolled in studies (10). These include:

\section{The procedure to be followed in the study,}

2.The benefits for the individual,

3.The discomforts and risks that are reasonably expected,

4.The alternative methods of therapy,

5.The willingness of the investigator to answer inquiries,

6.The right to refuse or to withdraw from the study without prejudice.

It is difficult to include patients for whom the best treatment is uncertain, even though the risk is quite minor. Of course, the value of proven therapies must not be overestimated, nor that of the placebo underestimated. In many cases, the placebo has an appreciable effect, particularly in the treatment of anxiety, depression or even a gastroduodenal ulcer. The problem can be raised by even temporary administration of drugs capable of causing teratogenic risks. It is particularly difficult to include patients with good equilibrium for a chronic disease who would be required to stop an effective treatment. This is the case notably for schizophrenic patients for whom achievement of therapeutic equilibrium is often a long process. However, failure to include schizophrenics resistant to current therapies would introduce a considerable bias for the development of new drugs. In fact, all of the staff members taking part in a trial must recommend inclusion and exclusion criteria that are specific, sensitive and reproducible [11].

A last problem concerns patients lost to view. In fact, this concept should not be applied to therapeutic studies insofar as a patient lost to view is considered as "dead" for purposes of statistical analysis. It is absolutely essential for an investigator to know his patients perfectly well. If the patient does not show up for a visit, it should be possible to reach him easily by telephone and ask why he does not wish to continue his treatment. This notion of a drop-out must not be confused with the requirement to exclude a participating patient after the trial has started.

\section{Conclusion}

Any clinical trial requires that the disease definition is well-specified; that patient eligibility is explicit; that the observation times be explicit; that the duration and endpoint of therapy be specified; that the duration of subsequent followup observation is specified; and that the unit of observation is defined. In a chronic disease, the potential biases that can readily be introduced by self-selection of patients who enter the trial and/or who return for subsequent observation become more important, because subjects are required to remain on treatment and/or observation for prolonged periods. Further, the cyclical nature of some chronic diseases may require special attention to baseline definitions of active disease and disease outcome.

Causal mechanism of relationship between the clinical outcome (efficacy or safety endpoints) and putative biomarkers, clinical baseline, and related predictors is usually unknown and must be deduced empirically from experimental data. Such relationships enable the development of tailored therapeutics and implementation of a precision medicine strategy in clinical trials to help stratify patients in terms of disease progression, clinical response, treatment differentiation, and so on [12].

The realization that bias in patient selection may influence the results of clinical studies has helped to establish the randomized controlled clinical trial in medical research. However, bias can be equally important at other stages of a trial, especially at the time of analysis. Withdrawing patients from consideration in the analysis because of ineligibility on account of study entry criteria, lack of compliance to the protocol or data of poor quality may be a source of systematic error.

There is no consensus on the appropriate use of patient selection factors. Some commentators believe that the efficiency and credibility of a trial are improved by making the population under study as narrowly defined as possible in order to reduce heterogeneity [13].

\section{References}

1. Begg CB. Selection of patients for clinical trials.Semin. Oncol. 1988;15(5):434-440.

2. Compston A. Selection of patients for clinical trials. Neuroepidemiology. 1987;6(1-2):34-39.

3. Langley GR, Sutherland HJ, Wong S, Minkin S, Llewellyn-Thomas HA, Till JE. Why are (or are not) patients given the option to enter clinical trials? Control. Clin. Trials. 1987;8(1):49-59.

4. Diamond S, Solomon GD, Freitag FG, Mehta N. Selection of patients-critical aspects. Neuroepidemiology. 1987;6(4):172-177.

5. Targum SD, Pollack MH, Fava M. Redefining affective disorders: relevance for drug development. CNS Neurosci Ther. 2008;14(1):2-9.

6. Wittes J, Lakatos E, Black D, Geraci B, Davis B, Moyé L, etc. Selecting screening criteria for clinical trials: an example from the Systolic Hypertension in the Elderly Program Control. Clin Trials. 1999;20(2):121-132.

7. Rajadhyaksha V. Conducting Feasibilities in Clinical Trials: An Investment to Ensure a Good Study. Perspect Clin Res. 2010;1(3):106109. 
8. Watson JM, Torgerson DJ. Increasing recruitment to randomised trials: a review of randomised controlled trials. BMC Med Res Methodol. 2006;6:34.

9. Prout TE. The ethics of informed consent. Control Clin Trials. 1981;1(4):429-434.

10. Stein RE, Jessop DJ. An ethics committee to aid in implementing a randomized clinical trial. Control Clin Trials. 1983;4(1):37-42.

11. Yusuf S, Held P, Teo KK, Toretsky ER. Selection of patients for randomized controlled trials: implications of wide or narrow eligibility criteria. Stat Med. 1990;9(1-2):73-83.

12. Huang X, Sun Y, Trow P, Chatterjee S, Chakravartty A, Tian L, et al. Patient subgroup identification for clinical drug development. Stat Med. 2017;36(9):1414-1428.

13. White D, Hind D. Projection of participant recruitment to primary care research: a qualitative study. Trials. 2015;16:473. doi: 10.1186/ s13063-015-1002-9 